\title{
Restoration of synaptic function in sight for degenerative retinal disease
}

\author{
Timm Schubert ${ }^{1}$ and Bernd Wissinger ${ }^{2}$ \\ ${ }^{1}$ Werner Reichardt Centre for Integrative Neuroscience (CIN) and Institute for Ophthalmic Research, and 2Molecular Genetics Laboratory, Institute for Ophthalmic Research, \\ University of Tübingen, Tübingen, Cermany.
}

\begin{abstract}
Synaptic disorganization is a prominent feature of many neurological diseases of the CNS, including Parkinson's disease, intellectual development disorders, and autism. Although synaptic plasticity is critical for learning and memory, it is unclear whether this innate property helps restore synaptic function in disease once the primary cause of disease is abrogated. An answer to this question may come from a recent investigation in $\mathrm{X}$-linked retinoschisis, a currently untreatable retinopathy. In this issue of the $J C l, O u$, Vijayasarathy, and colleagues showed progressive disorganization of key functional elements of the synapse between photoreceptors and $\mathrm{ON}$-bipolar cells in a retinoschisin-deficient mouse model. Moreover, they demonstrated that adeno-associated virus-mediated (AAV-mediated) delivery of the retinoschisin gene restores structure and function to the photoreceptor to ON-bipolar cell synapse in mouse models, even in adults at advanced stages of the disease. The results of this study hold promise that AAV-based supplemental gene therapy will benefit patients with $\mathrm{X}$-linked retinoschisis in a forthcoming clinical trial.
\end{abstract}

\section{X-linked retinoschisis: a} disease of synapse dysfunction $\mathrm{X}$-linked retinoschisis (XLRS) is a retinal degenerative disease leading to early visual loss. Morphologically, the disease is characterized by splitting through retinal layers and formation of intraretinal fluid-filled cystic cavities that are most prominent in the foveomacular region. XLRS is caused by mutations in the retinoschisin-encoding gene (RS1), which is mainly expressed in photoreceptors in the mature retina. Retinoschisin is a secreted protein that adopts an octameric structure and is a prominent component of the extracellular matrix (ECM) of both the photoreceptor inner segment and the retinal outer plexiform layer, where it may act as a cell-adhesion molecule. Although some interaction partners have been identified, the precise function of retinoschisin is largely unknown. A hallmark of XLRS is a reduction of the $b$-wave amplitude in light-evoked electroretinography (ERG) responses, which points to a principal defect in light-dependent signal transmission from photoreceptors to depolarizing ON-bipolar cells (1). In this issue, Ou, Vijayasarathy, and colleagues used $R s 1^{-/}$ knockout mouse model to study XLRSassociated alterations of the rod photoreceptor-bipolar cell synapse in detail (2).

In the retina, substantial synaptic remodeling has been described at the very first synapse of the visual system in several mouse degeneration models. In particular, the postsynaptic side of bipolar cells has been studied in great detail. Various degeneration-induced synaptic alterations have been observed, including sprouting of bipolar cell dendrites (3), downregulation of the metabotropic glu-

Related Article: p. 2891

Conflict of interest: The authors have declared that no conflict of interest exists.

Reference information: J Clin Invest. 2015;125(7):2572-2575. doi:10.1172/JCI82577.

tamate receptor (mGluR6)(4), alteration of downstream signaling cascade proteins (G proteins, nyctalopin, and transient receptor potential melastatin subfamily M member 1 [TRPM1]) (5), and compensatory expression of ionotropic glutamate receptors (6) and glutamate transporters (7). Interestingly, photoreceptor loss can affect glutamate receptor expression within a time span of a few hours, indicating a high degree of plasticity in the outer degenerative retina (8).

\section{Murine XLRS model provides insight into pathosynaptic phenotypes}

The extent and type of pathosynaptic alterations in retinal disease strongly depend on how degeneration affects the photoreceptor. For example, when rod photoreceptors vanish - as is the case in $r d 1$ and $r d 10$ mouse models - rod bipolar cells retract their dendrites, but express alternative somatic glutamate receptors to integrate glutamatergic input from remnant cones $(4,6,7)$. Conversely, if only glutamate release from the presynapse is reduced, but the rod itself is maintained, ectopic synapses between rods and rod bipolar cells are formed and mGluR6 distribution is strongly altered (reviewed in ref. 9). In this issue of the JCI, Ou, Vijayasarathy, and colleagues report a subtle pathosynaptic phenotype in a mouse model for XLRS. Using $R s 1^{-/-}$mice, these authors present evidence that proteins downstream of the intracellular signaling cascade are dislodged from postsynaptic bipolar cell dendrites; however, the glutamate-binding receptor mGluR6 itself remains at this site (Figure 1). Notably, compared with other murine retinal degeneration models, presynaptic structures were maintained, but the calcium levels in the presynaptic terminals were seemingly reduced in $\mathrm{Rs}^{-/}$mice. Moreover, expression of the glutamate transporter vGluT1 was reduced in the outer retina of $\mathrm{RsI}^{-/-}$, pointing to a 
A Rods

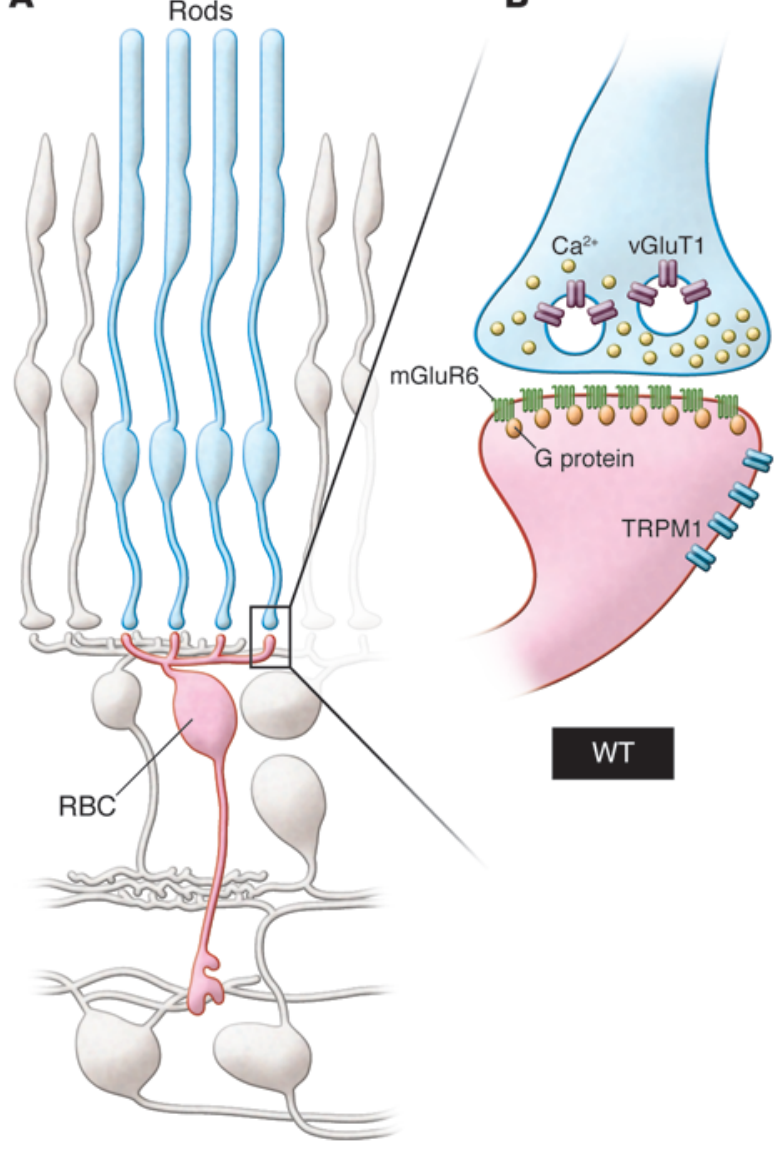

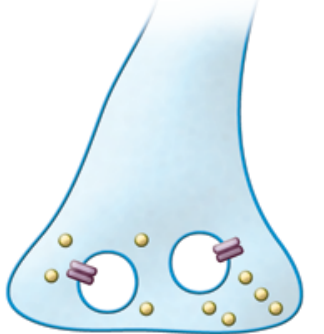

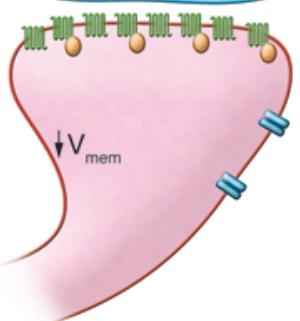

$R s 1^{-1}$ strong alteration of the glutamate release at the photoreceptor-bipolar cell synapse. When synaptic transmission is reduced, postsynaptic neurons normally respond in one of two possible ways. They either increase postsynaptic receptor density in response to the reduced level of transmitter to adjust the synaptic efficiency or they reduce the number of postsynaptic receptors to strengthen other connections. The compensatory pathway chosen very much depends on the specificity of the synapse and the overall activity level (reviewed in ref. 10). Surprisingly, the decreased calcium level in the $R s 1^{-/-}$photoreceptor terminals and the expected downregulation of glutamate release did not directly alter expression or localization of the glutamate-binding mGluR6 receptor on the dendrite tips; however, the calcium decrease reduced expression of downstream signaling molecules, including the cation channel TRPM1, subsequently leading to homeostatic hyperpolarization of the bipolar cells. Unlike earlier studies $(11,12)$, the study by $\mathrm{Ou}$, Vijayasarathy, and colleagues suggests that expression levels of
mGluR6 and TRPM1 are differently regulated. In this view, the distinct pathosynaptic patterns of the mGluR6-signaling cascade proteins observed in different mouse retinal degeneration models suggest that the postsynaptic macromolecular organization in bipolar cells depends on highly complex protein expression, trafficking, and sorting processes. However, a much more complete picture of the composition and regulation of the photoreceptor-bipolar cell synapse needs to be established to fully understand the pathosynaptic phenotype in $\mathrm{Rs}^{-/-}$animals. For example, further ultrastructural studies are required to evaluate fine-scale morphological alterations, similar to what has been performed at the photoreceptor synapse to study the ECM protein pikachurin (13). Additionally, it is not clear how glutamate release from photoreceptors is changed in regard to degenerative downstream processes. For example, in the $R s 1^{-/-}$retina, the calcium concentration in photoreceptor axon terminals is lower, but the expression levels of proteins that remove glutamate from the synaptic cleft (e.g., glutamate transport-
Figure 1. Functional remodeling of the rod photoreceptor-to-rod bipolar cell synapse in the retinoschisin-deficient retina. (A) This schematic shows the synaptic connection between rod photoreceptors (blue, rods) and rod bipolar cells (red, RBC) in the outer retina. For simplicity, all other cell types are shown in gray. (B) Magnified synapse illustrates the differences in organization of the rod-to-rod bipolar cell synapse between WT and synaptically remodeled $R_{s} 1^{-/-}$mutant retinae (right). In the $R s 1^{-1-}$ animals, the presynaptic structure of the rod is intact, although the intracellular calcium channel concentration and the vGluT1 expression are decreased, suggesting lower abundance of synaptic vesicles and reduced glutamate release. Note that the density of mGluR6 in the postsynaptic $R s 1^{-1-} \mathrm{RBC}$ resembles that in the WT RBC, whereas $G$ protein and TRPM1 channel expression at the dendritic tip are reduced.

ers) remain unclear, which in theory would allow the glutamate concentration in the synaptic cleft to be decreased or increased. A recently developed, genetically encoded glutamate-sensing indicator (14) has been successfully expressed in retinal cells to measure glutamate in the synaptic cleft; however, this sensor has not been used to evaluate glutamate in murine models of degeneration. Adeno-associated virusmediated (AAV-mediated) expression of such a bioindicator in bipolar cells to monitor glutamate release from photoreceptors has the potential to substantially advance our understanding of how altered glutamatergic drive from degenerating photoreceptors affects postsynaptic structures. As there is a high degree of plasticity at the first synapse of the mouse visual system in degeneration mouse models, a finding that presumably translates to patients, therapeutic intervention to rescue function of this important synapse may be feasible in the future.

\section{Gene therapy restores visual acuity in adult retinoschisin- deficient mice}

Currently, there is no effective treatment for patients with XLRS. Topical administration of carbonic anhydrase inhibitors ameliorates prominent morphological distortions, such as central macular thickness and the size of macular cysts; however, improvement of visual acuity upon treatment is controversial $(15,16)$. In contrast, in the $R s 1^{-/-}$mouse model for XLRS, supplemental AAV vector-mediat- 
ed gene therapy has not only been shown to reduce retinal swelling and cyst formation, but also to reduce photoreceptor loss and improve light-induced b-wave amplitude in ERG recordings (17-21). $\mathrm{Ou}$, Vijayasarathy, and colleagues have now substantiated previous reports that AAV-based gene delivery functionally rescues the visual defect by showing that reconstitution of retinoschisin expression ameliorates synaptic pathology by restoring presynaptic $\mathrm{Ca}^{2+}$ levels and vGlutT1 expression, postsynaptic organization of signal transduction components, and bipolar cell membrane potential (2). Importantly, these results were achieved in adult animals in which the pathology had already substantially progressed, indicating that function is regained through innate plasticity of the photoreceptor-toON-bipolar cell synapse.

Current state-of-the-art gene therapy for retinal dystrophies is surgically demanding and requires local detachment of the photoreceptor layer from the underlying retinal pigment epithelium for injection of viral vectors into the subretinal space (22-24). This transient ablation of the neuroretina poses additional procedural risks, such as retinal rupture, particularly in patients with a thinned, degenerating retina as the result of retinal dystrophies, and damage to the fovea, which increases the risk of compromising any residual central vision (25). Furthermore, subretinal injection only allows gene transduction at the site of injection and in parts of the retina that immediately surround the injection site. An alternative approach that overcomes these limitations is delivery of viral vectors through injection into the vitreous. This route of injection is a routine and safe procedure in ophthalmic surgery that is performed under local anesthesia. While penetration of AAV vectors from the vitreous into the outer retinal layers has been a major obstacle in early studies, recent capsid modifications have yielded vectors with improved ability to efficiently transduce outer retinal cell types, including photoreceptors and bipolar cells in the mouse retina $(26,27)$. It has yet to be shown whether penetration and efficacy of outer retinal cell transduction after intravitreal injection will show comparable results in the human retina and whether this route of injection will be suffi- cient for therapeutic success. In XLRS, the swelled and loosened retinal structure of this pathology may facilitate penetration of the therapeutic vector and transduction of photoreceptors and bipolar cells upon intravitreal injection. Prior studies with intravitreal injection of an AAV8 vector that is similar to that performed by $\mathrm{Ou}$, Vijayasarathy, and colleagues have shown that penetration into the outer retina of WT mice is much less effective than it is in the $R s 1^{-/-}$mutant retina, where the AAV8 vector readily transduces photoreceptors and rescues function (20). As retinoschisin is a secreted protein, could AAV-mediated expression of retinoschisin in other, more easily accessible retina cell types do the job? Likely not, as targeted expression of retinoschisin in Muller glial cells has been shown to be much less effective than expression in photoreceptors for rescuing defects in the $R s 1^{-/-}$mouse (21).

In conclusion, $\mathrm{Ou}, \mathrm{Vijayasarathy,} \mathrm{and}$ colleagues provide a body of work that supports other preclinical evidence that XLRS may be an excellent target for retinal gene therapy. Not only does innate synaptic plasticity restore structure and function in $\mathrm{Rs}^{\mathrm{T}^{--}}$mice, but the pathology of this disease provides advantageous access to outer retina layers for the intravitreal route delivery of therapeutic AAV vectors. The feasibility of this approach in patients is being evaluated in a phase I/IIa, prospective, three-dose escalation study that is currently underway (ClinicalTrials. gov NCT02317887) to probe safety and efficacy of gene therapy in XLRS patients.

\section{Acknowledgments}

The authors wish to acknowledge support of their work by the Charlotte and Tistou Kerstan Stiftung (RD-CURE to B. Wissinger), a grant from the German Ministry for Education and Research (BMBF; IonNeuroNet, grant no. 01GM1105A to B. Wissinger), and by the Deutsche Forschungsgemeinschaft (EXC 307, CIN and SCHU 2243/3-1 to T. Schubert).

Address correspondence to: Bernd Wissinger, Molecular Genetics Laboratory, Institute for Ophthalmic Research, University of Tübingen, Röntgenweg 11, D-72076 Tübingen, Germany. Phone: 49.7071.2985032; E-mail: wissinger@ uni-tuebingen.de.
1. Molday RS, Kellner U, Weber BH. X-linked juvenile retinoschisis: clinical diagnosis, genetic analysis, and molecular mechanisms. Prog Retin Eye Res. 2012;31(3):195-212.

2. Ou J, et al. Synaptic pathology and therapeutic repair in adult retinoschisis mouse by AAV-RS1 transfer. J Clin Invest. 2015;215(7):2891-2903.

3. Chang B, et al. The nob2 mouse, a null mutation in Cacna1f: anatomical and functional abnormalities in the outer retina and their consequences on ganglion cell visual responses. Vis Neurosci. 2006;23(1):11-24.

4. Barhoum R, et al. Functional and structural modifications during retinal degeneration in the rd10 mouse. Neuroscience. 2008;155(3):698-713.

5. Križaj D, Huang W, Furukawa T, Punzo C, Xing W. Plasticity of TRPM1 expression and localization in the wild type and degenerating mouse retina. Vision Res. 2010;50(23):2460-2465.

6. Chua J, Fletcher EL, Kalloniatis M. Functional remodeling of glutamate receptors by inner retinal neurons occurs from an early stage of retinal degeneration. J Comp Neurol. 2009;514(5):473-491.

7. Haq W, Arango-Gonzalez B, Zrenner E, Euler T, Schubert T. Synaptic remodeling generates synchronous oscillations in the degenerated outer mouse retina. Front Neural Circuits. 2014;8:108.

8. Dunn FA. Photoreceptor ablation initiates the immediate loss of glutamate receptors in postsynaptic bipolar cells in retina. J Neurosci. 2015;35(6):2423-2431.

9. D'Orazi FD, Suzuki SC, Wong RO. Neuronal remodeling in retinal circuit assembly, disassembly, and reassembly. Trends Neurosci. 2014;37(10):594-603.

10. Okawa H, Hoon M, Yoshimatsu T, Della Santina $\mathrm{L}$, Wong RO. Illuminating the multifaceted roles of neurotransmission in shaping neuronal circuitry. Neuron. 2014;83(6):1303-1318.

11. Xu Y, Dhingra A, Fina ME, Koike C, Furukawa T, Vardi N. mGluR6 deletion renders the TRPM1 channel in retina inactive. J Neurophysiol. 2012;107(3):948-957.

12. Cao Y, Posokhova E, Martemyanov KA. TRPM1 forms complexes with nyctalopin in vivo and accumulates in postsynaptic compartment of ON-bipolar neurons in mGluR6-dependent manner. JNeurosci. 2011;31(32):11521-11526.

13. Sato S, et al. Pikachurin, a dystroglycan ligand, is essential for photoreceptor ribbon synapse formation. Nat Neurosci. 2008;11(8):923-931.

14. Marvin JS, et al. An optimized fluorescent probe for visualizing glutamate neurotransmission. Nat Methods. 2013;10(2):162-170.

15. Genead MA, Fishman GA, Walia S. Efficacy of sustained topical dorzolamide therapy for cystic macular lesions in patients with $\mathrm{X}$-linked retinoschisis. Arch Ophthalmol. 2010;128(2):190-197.

16. Khandhadia S, Trump D, Menon G, Lotery AJ. $\mathrm{X}$-linked retinoschisis maculopathy treated with topical dorzolamide, and relationship to genotype. Eye (Lond). 2011;25(7):922-928.

17. Zeng Y, et al. RS-1 Gene Delivery to an adult rs1h knockout mouse model restores ERG b-wave with reversal of the electronegative waveform of $\mathrm{X}$-Linked retinoschisis. Invest Ophthalmol Vis Sci. 
2004;45(9):3279-3285.

18. Min SH, et al. Prolonged recovery of retinal structure/function after gene therapy in an Rs1h-deficient mouse model of $\mathrm{x}$-linked juvenile retinoschisis. Mol Ther. 2005;12(4):644-651.

19. Janssen A, et al. Effect of late-stage therapy on disease progression in AAV-mediated rescue of photoreceptor cells in the retinoschisin-deficient mouse. Mol Ther. 2008;16(6):1010-1017.

20. Park TK, et al. Intravitreal delivery of AAV8 retinoschisin results in cell type-specific gene expression and retinal rescue in the Rs1-KO mouse. Gene Ther. 2009;16(7):916-926.
21. Byrne LC, et al. Retinoschisin gene therapy in photoreceptors, Müller glia or all retinal cells in the $\mathrm{Rs}^{\mathrm{h}} \mathrm{h}^{-/}$mouse. Gene Ther. 2014;21(6):585-592.

22. Maguire AM, et al. Safety and efficacy of gene transfer for Leber's congenital amaurosis. $N$ Engl JMed. 2008;358(21):2240-2248.

23. Bainbridge JW, et al. Effect of gene therapy on visual function in Leber's congenital amaurosis. N EnglJ Med. 2008;358(21):2231-2239.

24. MacLaren RE, et al. Retinal gene therapy in patients with choroideremia: initial findings from a phase $1 / 2$ clinical trial. Lancet.
2014;383(9923):1129-1137.

25. Jacobson SG, et al. Gene therapy for leber congenital amaurosis caused by RPE65 mutations: safety and efficacy in 15 children and adults followed up to 3 years. Arch Ophthalmol. 2012;130(1):9-24.

26. Kay CN, et al. Targeting photoreceptors via intravitreal delivery using novel, capsid-mutated AAV vectors. PLoS One. 2013;8(4):e62097.

27. Dalkara $D$, et al. In vivo-directed evolution of a new adeno-associated virus for therapeutic outer retinal gene delivery from the vitreous. $\mathrm{Sci}$ Transl Med. 2013;5(189):189ra76. 